\title{
Analisis Organizacional Y Financiera Del Uso De Prácticas Sustentables En Empresas Industriales Del Municipio De Tulancingo De Bravo Perteneciente Al Estado De Hidalgo ${ }^{1}$
}

Dr. Sergio Demetrio Polo Jiménez

Dr. Eleazar Villegas González

Dr. Tirso Javier Hernández Gracia

Dr. Danae Duana Ávila

Dra. Ma. Del Rosario García Velázquez

Universidad Autónoma del Estado de Hidalgo, México

doi: 10.19044/esj.2017.v13n15p300 URL:http://dx.doi.org/10.19044/esj.2017.v13n15p300

\begin{abstract}
The purpose of the present investigation is to identify those internal factors that influence the implementation of socially responsible practices (energy saving) in a sample of 5 industrial companies in the municipality of Tulancingo de Bravo, Hidalgo in 2016. To achieve the proposed objectives, an index similar to that proposed by Barrera et al. (2004), Sepúlveda et al. (2002); Vázquez et al. (2011); Céspedes (2011); Carrillo in (2014). The results of the application of the Kruskal-Wallis test, could allow us to affirm that the development of sustainable practices in the companies of the sample, are still far from those carried out in Europe.
\end{abstract}

Keywords: Sustainability, Sustainability Index, Linear Regression, Energy Saving Practices

\section{Resumen}

El propósito de la presente investigación, se orienta en identificar aquellos factores internos, que influyen para la realización de prácticas socialmente responsables (ahorradoras de energía eléctrica), en una muestra de 5 empresas industriales del municipio de Tulancingo de Bravo, Hidalgo

${ }^{1}$ Se agradece la colaboración en la realización del presente trabajo de investigación de los alumnos de la Licenciatura en Contaduría de la Universidad Autónoma del Estado de Hidalgo, Martín Benítez Daniela y Marco Antonio Aparicio Ramírez 
en el año 2016. Para alcanzar los objetivos propuestos, se desarrolla un índice similar al propuesto por Barrera et al. (2004), Sepúlveda et al. (2002); Vázquez et al. (2011); Céspedes (2011); Carrillo en (2014). Los resultados de la aplicación del Test de Kruskal Wallis, nos podrían permitir afirmar que el desarrollo de prácticas sustentables en las empresas de la muestra, aún están lejos de aquellas llevadas a cabo en Europa.

Palabras clave: Montgaudier, Minéraux lourds, Minéraux légers, Minéraux argileux, Kaolinite, Tardoire, Charente, France

\section{Introduction}

El tema del cuidado y protección al medio ambiente, ha tomado mayor importancia en los últimos años, esto debido a los problemas de contaminación y calentamiento global que han ido en aumento. El desarrollo de actividades sustentables, en los sectores público y privado, resulta trascendental para la preservación de la biodiversidad y se convierte en una variable estratégica, ya que las empresas aparecen de forma simultánea, como causantes del problema medioambiental y como parte de la solución, al tener $\mathrm{y}$ deber conciliar patrones de producción y de consumo (Brio $\mathrm{y}$ Junquera, 2001).

De esta manera, uno de los puntos a tratar en las agendas de los organismos reguladores del medio ambiente alrededor del mundo, es el tema de la sustentabilidad, como lo establece, la Organización de las Naciones Unidas (ONU), a través del Informe Brundtland de la Comisión Mundial sobre Medio Ambiente y Desarrollo (CMMAyD), donde se pronuncia por vez primera sobre la definición de sustentabilidad como el desarrollo humano, que satisface las necesidades del presente sin comprometer la capacidad de las generaciones futuras, o el Consejo Mundial de la Energía (CME), institución también, reconocida por la (ONU), encargada de la promoción y el uso sostenible de la energía. El CME, organizada la Cumbre Mundial de Líderes de Energía, espacio que busca facilitar un diálogo permanente de alto nivel sobre asuntos críticos que afectan al mundo de la energía.

En México, la preocupación del cuidado a la biodiversidad, ha rendido frutos y cada día aumenta el número de empresas con un claro compromiso hacia la sustentabilidad, utilizando tecnologías ecoeficientes, tal es el caso de la Bolsa Mexicana de Valores que en 2011, crea el Índice de Sustentabilidad, conformado por 28 empresas que han sido calificadas principalmente por tres criterios: manejo y uso de los recurso naturales, responsabilidad social y gobierno corporativo. Para las PyMES, estas prácticas no son muy comunes, pues con facilidad se confunden los 
términos, lo que lleva a la mala implementación de sus actividades sustentables.

En este sentido, el presente trabajo, está encaminado en analizar si las prácticas ahorradoras de energía eléctrica desarrolladas por una muestra de empresas industriales del municipio de Tulancingo, Hidalgo difieren de las propuestas en un modelo de gestión energético español, situación que permita, la creación de un modelo de gestión de energía eléctrica, que coadyuve a la disminución del consumo de energía, reducir costos, al aumento del compromiso social y las finanzas corporativas.

Para alcanzar los objetivos propuestos, se estructura el estudio de la siguiente manera: tras la introducción, el trabajo dedica el siguiente apartado a la revisión de la literatura previa y planteamiento de hipótesis; posteriormente se da a conocer la metodología aplicada, describiendo la muestra y definiendo las variables objeto de estudio; en el cuarto apartado describimos los resultados obtenidos y finalizamos con la exposición de las principales conclusiones e implicaciones.

\section{Revision De La Literatura}

Es abundante la literatura, que trata de conocer la relación que existe entre determinados factores organizacionales (internos y externos) y la responsabilidad social organizacional, a través del desarrollo de prácticas en favor de la conservación ambiental. Por su importancia, destacan entre otros, el realizado por Barroso (2008), quien busca identificar las acciones de Responsabilidad Social Corporativa (RSE), que llevan a cabo en 40 empresas ubicadas en la ciudad de Mérida Yucatán. Mediante una guía de entrevistas semi estructuradas, sus resultados le permiten afirmar que en ningún caso, se cumple con el concepto de RSC en su totalidad, por lo que no pueden llamarse estas compañías, socialmente responsables.

El trabajo presentado por Milanés et al. (2012), nos refiere a los avances de sostenibilidad en la actividad turística, donde se analiza una muestra formada por 69 hoteles cotizados europeos, con el propósito de conocer la relación existente entre la rentabilidad empresarial y el desempeño medioambiental. A través de la aplicación del método de Ward (análisis de clúster jerárquico), se obtuvo que la mayoría de los hoteles observados, carece de una política de protección medioambiental, lo que influye significativamente en sus resultados financieros.

De la misma manera, Alonso et al. (2012) estudian a 19 empresas que representan el $15 \%$ de las que cotizan en la BMV, con el objeto de analizar el desempeño financiero y la RSE, mediante un modelo de regresión lineal, sus resultados muestran, que existe (al igual que Milanés et al. 2012), una relación de tipo positivo entre el desempeño financiero y la Responsabilidad Social Empresarial. 
Tres años más tarde y siguiendo con compañías listadas, el estudio de Morales (2015), referente al desempeño financiero y la RSE, mediante la aplicación de análisis multivariantes, sus resultados demostraron que las empresas reconocidas por el mercado financiero como sustentables, tienen los valores más altos de rentabilidad frente a las que no son reconocidas, para ello, analiza una muestra formada por 30 empresas del sector industrial cotizadas en la Bolsa Mexicana de Valores (BMV).

El estudio realizado por Reza-Torres (2015), se orienta en identificar las variables demográficas que condicionan el consumo de energía eléctrica, en los municipios del Estado de Jalisco, México. Sus resultados le permiten afirmar que los modelos habitacionales en donde se fomenta la organización social, aportan a la reducción del consumo de electricidad, respecto del modelo de organización en donde se da la fragmentación de los habitantes.

Existen otros estudios, encaminados a identificar si el desarrollo de prácticas socialmente responsables (específicamente aquellas relacionadas con el cuidado al medio ambiente) mantienen alguna influencia en el performance de la empresa, para ello, aplican índices de sustentabilidad como lo indican los trabajos realizados por Barrera et al. (2004) que valora la sustentabilidad de las actividades y procesos asociados a la refinación de petróleo crudo; Sepúlveda et al. (2002), que establece la metodología para medir el desarrollo sostenible territorial costarricense; Vázquez et al. (2011), que analiza las actividades sustentables de empresas dedicadas a la producción de ganado en Cuba o el trabajo realizado por Carrillo en 2014 que analiza el grado de sustentabilidad de conjuntos urbanos del Estado de México.

\section{Diseño Y Metodoloogia}

Con el propósito de identificar, las actividades de ahorro de energía eléctrica realizadas por las empresas de la muestra, se aplica el instrumento de buenas prácticas para la reducción del consumo energético y responsabilidad social, propuesto por el Observatorio español de la industria del sector textil y de la confección, (2011), con la intención de generar alternativas que permitan el consumo responsable de energía eléctrica y aumente el compromiso organizacional en materia de responsabilidad social, influyendo significativamente en su rentabilidad.

\section{Variable Dependiente}

Atendiendo a la literatura previa y en virtud de que el instrumento de medición contiene información inherente a temas de ahorro de energía eléctrica, responsabilidad social y financiera, se construye un índice similar al presentado por Barrera et al. (2004), Sepúlveda et al. (2002); Vázquez et al. (2011); Céspedes (2011); Carrillo en (2014), llamado Índice de 
sustentabilidad y responsabilidad social y financiero (ISRSF), cuya expresión es la siguiente:

\section{$I S R S F=\sum R s i+R n o / \sum M P R$}

Donde:

ISRSF= Índice de sustentabilidad y Responsabilidad Social

$\mathrm{Rsi}=$ Respuestas con valor de 1 o respuesta "si"

$\mathrm{Rno}=$ Respuestas con valor de 0 o respuesta "no"

$\mathrm{MPR}=$ Máxima Puntuación por Respuesta

En una segunda parte, se buscara identificar aquellas actividades intrínsecas (Escolaridad del responsable organizacional, Experiencia en el extranjero, Experiencia en el sector) a la organización, que influyen significativamente en el ahorro de energía y el impacto social y financiero.

\section{VARIABLES INDEPENDIENTES}

La Tabla 1, muestra la definición de cada una de las variables independientes.

Tabla 1: Variables independientes

\begin{tabular}{|c|c|c|}
\hline Nombre & Expresión & Definición \\
\hline Edad del entrevistado & EDAD & $\begin{array}{l}\text { Variable de tipo categórico que toma el } \\
\text { valor de } 1 \text { cuando la edad se ubica de } 20 \text { a } \\
40 \text { años, } 2 \text { cuando la edad esta de } 41 \text { a } 60 \\
\text { años y } 3 \text { cuando es mayor de } 60 \text { años }\end{array}$ \\
\hline Grado de escolaridad del entrevistado & ESCOLARIDAD & $\begin{array}{l}\text { Variable de tipo categórico que toma el } \\
\text { valor de } 1 \text {, cuando el entrevistado presenta } \\
\text { estudios de secundaria, } 2 \text { bachillerato y } 3 \\
\text { para educación profesional }\end{array}$ \\
\hline $\begin{array}{l}\text { Experiencia en el extranjero del } \\
\text { entrevistado }\end{array}$ & EXP_EXT & $\begin{array}{l}\text { Variable de tipo dicotómico que toma el } \\
\text { valor de } 1 \text { para "si" y } 0 \text { para "no" }\end{array}$ \\
\hline Experiencia en el sector del entrevistado & EXP_SECTOR & $\begin{array}{l}\text { Variable categórica con tres valores, } 1 \\
\text { cuando se cuenta con experiencia de } 1 \text { a } 10 \\
\text { años, } 2 \text { de } 11 \text { a } 30 \text { años y } 3 \text { cuando la } \\
\text { experiencia del entrevistado es mayor de } 30 \\
\text { años }\end{array}$ \\
\hline
\end{tabular}

Fuente: Elaboración propia 


\section{Muestra}

La muestra está formada por 5 empresas industriales pertenecientes al municipio de Tulancingo de Bravo en el Estado de Hidalgo, se presenta el detalle de cada una de ellas en la (Tabla 2). Las empresas denominadas como empresa 4 y empresa 5 solicitaron no dar a conocer su nombre.

Tabla 2: Detalle de la muestra

\begin{tabular}{l|lcc}
\hline Nombre & Sector & Región & $\mathrm{N}^{\circ}$ empleados \\
\hline KY2 KYa2 & $\begin{array}{l}\text { Textil Punto } \\
\text { fino } \\
\text { Textil Punto } \\
\text { CREACIONES REGIS }\end{array}$ & $\begin{array}{c}\text { Tulancingo } \\
\text { Tulancingo }\end{array}$ & 60 \\
ABRAXAS & $\begin{array}{c}\text { Textil Punto } \\
\text { fino }\end{array}$ & Tulancingo & 25 \\
EMPRESA 4 & $\begin{array}{l}\text { Textil acabados } \\
\text { Textil acabados }\end{array}$ & $\begin{array}{c}\text { Tulancingo } \\
\text { Tulancingo }\end{array}$ & 20 \\
EMPRESA 5 & & & 30 \\
\hline
\end{tabular}

Fuente: Elaboración propia

\section{Estadísticos Descriptivos Del Indice De Ahorro De Energía Y Responsabilidad Social Y Financiero (Isrsf)}

Los resultados del análisis descriptivo del ISRSF, aplicado a la muestra de empresas industriales del municipio de Tulancingo de Bravo Hidalgo, en función del grado de escolaridad del responsable organizacional, muestran que las compañías que realizan el mayor número de actividades ahorradoras de energía, y que consideran que el impacto financiero es bueno o rentable, son aquellas cuyos responsables cuentan con una educación superior, con un valor medio superior al 50\% (Grafico 1).

Gráfico 1: Comportamiento del IAERS en función del grado educativo del responsable

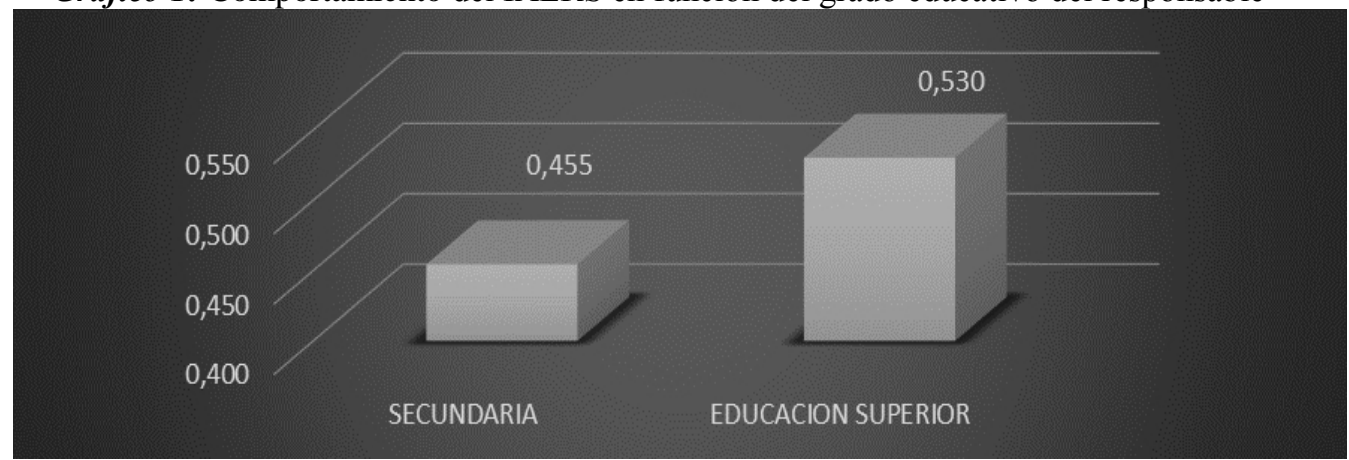

Fuente: Elaboración propia

Los valores del índice, respecto a la experiencia de haber realizado una estancia el extranjero por parte del responsable organizacional, se recoge en el Gráfico 2, donde se demuestra que la mayor cantidad de actividades sustentables con impacto financiero positivo, pertenecen a las compañías cuyos dirigentes no han salido del país, con un valor cercano al 56\%. 
Gráfico 2: Comportamiento del IAERS en función de la experiencia en el extranjero

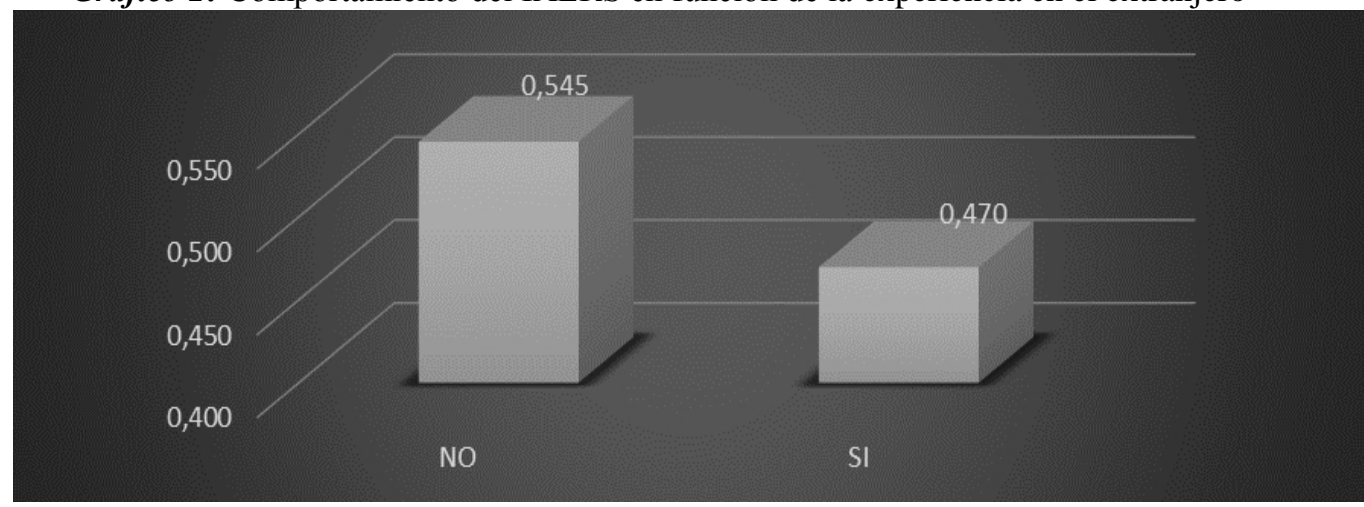

Fuente: Elaboración propia

Respecto a las compañías que cuentan con más de 30 años de experiencia en el sector, estas representan más del $51 \%$ que en mayor medida cuidan el medio ambiente y que consideran que sus prácticas socialmente responsables, apoyan en la generación de utilidades (Grafico 3).

Gráfico 3: Comportamiento del IAERS en función de la experiencia en el ramo

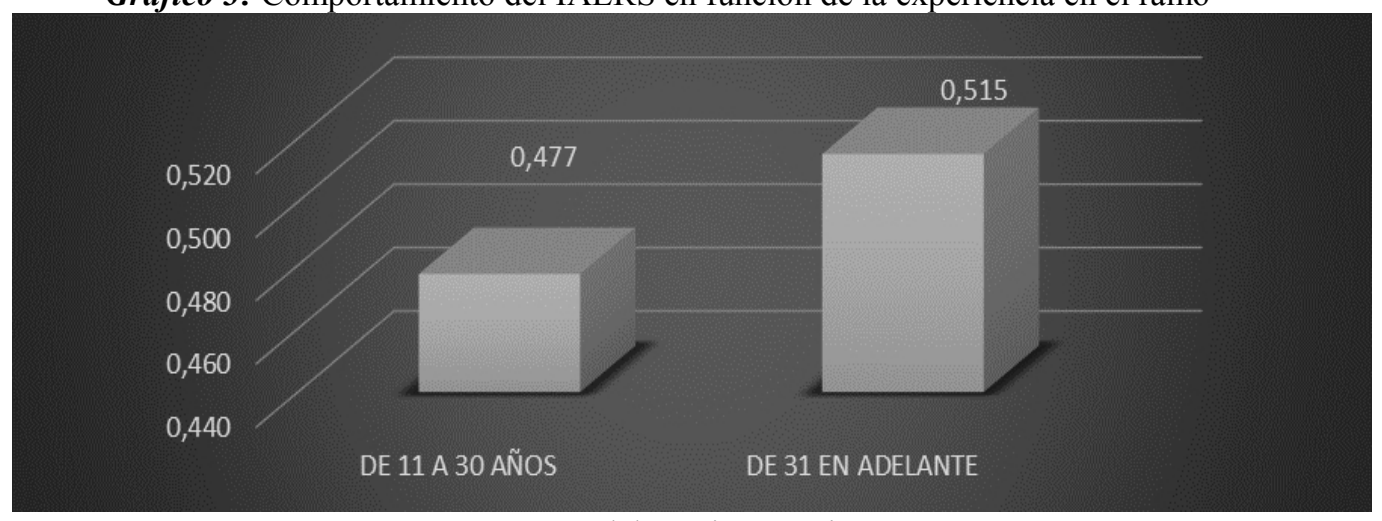

Fuente: Elaboración propia

\section{Técnicas De Análisis}

\section{Analisis Univariante}

Con el propósito de conocer si existe una relación estadísticamente significativa, entre el valor del ISRSF y determinadas características intrínsecas a las empresas de la muestra, en la selección de los tests a utilizar, debemos tener en cuenta los supuestos en los que se basan. A pesar del tamaño de la muestra, decidimos analizar la normalidad de las distribuciones para en su caso aplicar test paramétricos ya que, en general, la potencia de un test no paramétrico es menor que la de su homólogo paramétrico, excepto en contadas ocasiones (Tanizaki, 1997; Freidlin y Gastwirth, 2000). No obstante, los resultados muestran que los datos no persiguen una normalidad estadística, por tanto, se aplican pruebas no paramétricas (Test de Kruskall Wallis). 
Los resultados de la aplicación del Test de Kruskall Wallis, se recoge en la Tabla 3 donde podemos observar que la variable ESCOLARIDAD, mantiene una influencia significativa con el valor del ISRSF, lo que significa que el nivel de habilitación del responsable organizacional, condiciona la responsabilidad social corporativa, a través del uso de prácticas ahorradoras de energía eléctrica y la percepción de utilidades financieras.

La variable EXP_SECTOR, no es significativa. Por último, con un nivel de significatividad del 90\%, la variable EXP_EXT, es influyente en el valor del índice, lo que significa que el contar con alguna experiencia en el extranjero, apoya al cuidado del medio, por medio del uso de prácticas socialmente responsables orientadas a fortalecer sus finanzas.

Tabla 3: Niveles de influencia de las variables independientes respecto al ISRSF

\begin{tabular}{|c|r|c|}
\hline Variable & P-Value & Significatividad \\
\hline ESCOLARIDAD & 0.076 & $* *$ \\
\hline EXP_SECTOR & 0.717 & No \\
\hline EXP_EXT & 0.107 & ${ }^{*}$ \\
\hline
\end{tabular}

Significatividad $99 \% * * *, 95 \% * *$ y $90 \% *$

\section{Conclusiones}

La presente investigación tiene como objetivo, identificar las practicas socialmente responsables (en cuanto a ahorro de energía eléctrica), en una muestra de 5 empresas industriales del municipio de Tulancingo de Bravo perteneciente al Estado de Hidalgo, bajo el esquema de un modelo similar al por el Observatorio industrial textil y de la confección (2011).

Los resultados descriptivos, nos podrían permitir afirmar que el desarrollo de prácticas sustentables en las empresas de la muestra, aun están lejos de aquellas llevadas a cabo en Europa, pues el valor del índice por término medio, es inferior en todos los casos al $60 \%$. Se debe de reconocer, la necesidad de salir del país, a aquellos lugares donde hacen de su cultura, una verdadera filosofía de vida.

En definitiva, se propone que se establezcan modelos de gestión de responsabilidad social corporativa para todas las empresas de todos los sectores en el país y que exista un seguimiento muy cercano de las autoridades reguladoras en esta materia. Es necesaria, una mayor cultura de cuidado ambiental, la práctica de acciones sustentables en materia de ahorro genera beneficios financieros en las industrias, al tiempo que promueve la sustentabilidad.

\section{References:}

1. Alonso-Almeida, M.; Rodríguez, M.; Cortez, K.; Abreu, J. (2012): "La responsabilidad social corporativa y el desempeño financiero: un 
análisis en empresas mexicanas que cotizan en la bolsa", Contaduría y Administración, Vol. 57 No. 1, pp. 53-77

2. Barrera, A.; Saldivar, A.; Nava, M.; Ortíz, S.; Aguilar, S.; Villaseñor, E. (2004):'Índice de sustentabilidad industrial: Refinería Miguel Hidalgo", Problemas del Desarrollo, Vol- 35, No 137, pp 77-93

3. Barroso, F. (2008):" La responsabilidad social empresarial Un estudio en cuarenta empresas de la ciudad de Mérida, Yucatán"

4. Brío, J. A.; Junquera, B. (2002): "Medio ambiente y empresa: de la confrontación a la oportunidad", Tribuna de Economía, $\mathrm{N}^{\circ} 798$

5. Carrillo, A.; Jiménez, P.; Carreño, F. (2014):'ssistema de indicadores territoriales para evaluar la sustentabilidad de conjuntos urbanos en el estado de México: caso de estudio Calimaya", $21^{\circ}$ Encuentro Nacional sobre Desarrollo Regional en México. Mérida, Yucatán del 15 al 18 de noviembre de 2016. AMECIDER - ITM

6. Céspedes (2001): "Índice de sustentabilidad ambiental: sustentabilidad ambiental comparada en las entidades federativas de México. Centro de estudios del sector privado para el desarrollo sustentable, p. 220

7. Déniz, J. et al " Gestión ambientalmente responsable y valor de mercado de las acciones en situaciones de crisis financieras"

8. Freidlin, B y Gastwirth, J.L. (2000): "Change Point Tests Designed for the Analysis of Hiring Data Arising in Equal Employment Cases", Journal of Business and Economic Statistics, 18, pp. 315-322

9. Morales, J.A. (2015): "La acreditación de sustentabilidad y su impacto en los márgenes de rentabilidad del sector industrial de la Bolsa Mexicana de Valores", Respuestas, vol. 20, no. 1, pp. 16-29

10. Milanes, P.; Pérez, E.; Ortega, F. (2012): "Factores determinantes del nivel de compromiso medioambiental voluntario adquirido por los hoteles cotizados europeos", Contaduría y Administración. 57 (4), pp 197-233

11. Observatorio industrial del sector textil y de la confección (2011): Disponible http://www.minetur.gob.es/industria/observatorios/SectorTextil/Activ idades/2011/CIE,\%20FITAG-UGT,\%20FITEQA-

CCOO/Necesidades_Formacion_Parte_I.pdf, (Fecha de consulta: 10 de junio de 2016)

12. Reza-Torres, E. (2015): "Modelado del consumo de energía eléctrica residencial respecto de las dinámicas sociodemográficas en los municipios del estado de Jalisco utilizando la técnica de regresión lineal múltiple" Instituto Tecnológico y de Estudios Superiores de Occidente - Repositorio institucional ITESO. Proyecto de investigación aplicada 
13. Rodríguez, M.” La responsabilidad social corporativa y el desempeño financiero: un análisis en empresas mexicanas que cotizan en la bolsa"

14. Sepúlveda, S.; Chavarría, H.: Castro, A.; Rojas, P.; Picado, E.; Bolaños, D. (2002): "Metodología para estimar el nivel de desarrollo sostenible en espacios territoriales". IICA. https://ecaths1.s3.amazonaws.com/desarrollosostenible/176848813.M etodologia\%20e. (Fecha de búsqueda enero 2017)

15. Tanizaki, H., 1997. Power Comparison of Nonparametric Tests: Small Sample Properties from Monte-Carlo Experiments, Journal of Applied Statistics, 24 (5), pp.603-632

16. Vázquez, Y.; Guerra, C.; Sánchez, O. (2011):" Modelación Estadístico-Matemática para el estudio de la sostenibilidad socioeconómica en el sector agrícola-pecuario del municipio San José de las Lajas, provincia Mayabeque", Ciencias Técnicas Agropecuarias, Vol. 20, No4, pp 69-74 